\title{
O Uso do Teste de Escape do Balonete como Fator Preditor de Laringoespasmo*
}

\section{The Use of the Cuff Leak Test as a Factor to Predict Laryngospasm}

\author{
Lucas Montano Paternostro Saback', Gustavo Fernandes Vieira², Marcelo Dourado Costa ${ }^{3}$
}

\section{RESUMO}

JUSTIFICATIVA E OBJETIVOS: O desmame da ventilação mecânica é um desafio na prática da unidade de terapia intensiva (UTI) e está relacionado a diversas complicações. Uma dessas complicações relaciona-se ao laringoespasmo pós-extubação, evento que muitas vezes pode ser previsto através do teste de escape do balonete (TEB). O objetivo deste estudo foi demonstrar que o TEB é um método simples, confiável e de baixo custo para avaliar o grau de obstrução de via aérea superior em pacientes no processo de desmame da ventilação mecânica.

CONTEÚDO: Foi realizada revisão sistemática da literatura através das bases de dados MedLine, SciElo e LILACS com publicações entre 1995 e 2007. Os artigos incluídos abordaram o uso do teste do balonete como índice preditivo para laringoespasmo e falha na extubação traqueal. Não fizeram parte estudos com animais e revisões bibliográficas. Procurou-se relacionar o TEB com tempo de ventilação mecânica, idade e grupos específicos de pacientes que se beneficiaram com a técnica.

CONCUSÕES: O TEB pode ser considerado bom índice preditivo para identificar a presença de laringoespasmo pós-extubação, quando levadas em considera-

1. Fisioterapeuta, Hospital Santa Izabel

2. Fisioterapeuta, Hospital Salvador

3. Fisioterapeuta, Hospital São Rafael

*Recebido do Hospital Santa Izabel, Salvador, BA

Apresentado em 09 de maio de 2007

Aceito para publicação em 17 de dezembro de 2007

Endereço para correspondência:

Lucas Montano Paternostro Saback

R. Sócrates Guanaes Gomes, 215/704, Cidade Jardim

40296-720 Salvador, BA

Fone: (71) 3452-0458

E-mail: lucasmps@pop.com.br

(C)Associação de Medicina Intensiva Brasileira, 2008 ção as características da população estudada.

Unitermos: desmame da ventilação mecânica, edema laríngeo, falha na extubação, intubação traqueal, teste de escape do balonete

\section{SUMMARY}

BACKGROUND AND OBJECTIVES: Weaning patients from mechanical ventilation is a challenge in the intensive care unit (ICU) practice and is related to some complications. One of these is the pos-extubation laryngospasm, an event that can be anticipated for the cuff leak test (CLT). The objective was demonstrate that the CLT is a simple, reliable and low costs method to available the presence of obstruction in high airway in patients under weaning ventilator.

CONTENTS: It was made a systematic review in databases MedLine, SciElo and LILACS with articles from 1995 to 2007. The selected studies focused the use of the CLT to predict laryngospasm and extubation failure. It was excluded studies with animals and others literature reviews. It was looked correlates the CLT with mechanical ventilation's days, age and specific groups that could beneficiates with the application of this technique.

CONCLUSIONS: The CLT can be considerate a good index to predict laryngospasm pos-extubation, when considerate the studied population characteristics.

Key Words: cuff leak test, extubation failure, laryngeal edema, tracheal intubation, weaning from mechanical ventilation

\section{INTRODUÇÃO}

As falhas na extubação traqueal, com conseqüente necessidade de re-intubação, ocorrem em torno de $20 \%$ dos pacientes nas primeiras 24 a 72 horas da extubação planejada ${ }^{1}$. Muitos fatores aparecem como riscos de falha pós-extubação para os pacientes como idade superior a 70 anos, maior gravidade da doença de base no momento do desmame da ventilação mecânica, uso 
contínuo de sedação, tempo prolongado de ventilação mecânica e a presença de laringoespasmo ${ }^{1,2}$.

O laringoespasmo deve-se à inflamação e edema da região laríngea pela presença do tubo traqueal, tendo relação com o tempo de ventilação mecânica, sendo uma complicação freqüente (3\% a $22 \%$ ) durante o processo de desmame ${ }^{3,4}$. Dentre outras complicações da intubação traqueal pode-se inferir perda ciliar, ulcerações da via aérea, hemorragia, estenose subglótica, fístula traqueo-esofágica, além de formação de granulomas ${ }^{4-6}$.

A obstrução da via aérea alta secundária ao edema de laringe pode levar à diminuição do diâmetro interno da via aérea e sua influência na extubação pode ser avaliada através do teste de escape do balonete (TEB) ${ }^{3,4,7}$. Esse teste consiste na desinsuflação do balonete do tubo traqueal para verificação do grau de escape aéreo ao redor do tubo com mensuração do volume de ar (teste quantitativo) ou presença do estridor laríngeo (teste qualitativo), evidência clínica do laringoespasmo.

Outros métodos como a tomografia computadorizada, a ressonância nuclear magnética e a ultra-sonografia laríngea podem visualizar edema na via aérea superior, porém seu uso é restrito, por limitações das técnicas e 0 alto custo ${ }^{3,8}$.

O objetivo deste estudo foi demonstrar que o teste de escape do balonete é um método simples, confiável e de baixo custo, para avaliar o grau de obstrução de via aérea superior em pacientes no processo de desmame da ventilação mecânica.

\section{TIPOS DE TESTE}

A técnica de avaliação do escape aéreo foi descrita inicialmente, em sua forma qualitativa, por Fischer e Raper $^{9}$, em estudo envolvendo 62 pacientes. Quando o paciente estivesse apto para a extubação, deveria ser retirado do ventilador mecânico, desinsuflar o balonete, ocluir o tubo com um dedo e avaliar a presença ou não de escape de ar peritubular, usando um estetoscópio; estabelecendo como positivo para o escape quando se auscultasse o ruído da passagem do ar peritubular com o tubo traqueal ocluído.

No presente estudo foi observado que quando o teste era positivo para o escape, havia grande valor preditivo para ausência de laringoespasmo. No entanto, quando o teste era negativo muitos pacientes ainda assim não apresentavam estridor laríngeo, portanto não servia como critério para extubação, podendo ser responsável por intubação prolongada ou traqueostomia desnecessária.

Já a forma quantitativa descrita por Miller e Cole ${ }^{10}$ é a forma de avaliação mais utilizada, com pequena diferença de parâmetros entre os pesquisadores. $O$ paciente deve realizar satisfatoriamente um teste de autonomia em ventilação espontânea em CPAP de $5 \mathrm{cmH}_{2} \mathrm{O}$ por duas horas, depois modificar para o modo assistidocontrolado a volume (com volume-corrente inspirado a $10 \mathrm{~mL} / \mathrm{kg}$ ) e observado o volume-corrente (VC) expirado. Em seguida, desinsuflar totalmente o balonete e anotar a média dos três VC expirados mais baixos entre os seis primeiros ciclos respiratórios. $O$ valor de escape é a diferença entre o volume inspirado e a média do volume expirado, e sua respectiva porcentagem, tendo como ponto de corte para o escape $12 \%$ ou 110 a $130 \mathrm{~mL}$. Nesta forma de avaliação um fator determinante para o resultado do TEB é a área de secção transversa ao redor do tubo. No entanto, recentemente Prinianakis e col. ${ }^{3}$, afirmaram que além da área de secção transversa, a fase inspiratória do TEB é influenciada pela complacência do sistema respiratório e do fluxo inspiratório. A resistência do sistema respiratório influencia a fase expiratória do teste, havendo a possibilidade de alteração do valor do escape aéreo quando estas variáveis estiverem alteradas ${ }^{3,11}$.

\section{TEMPO DE INTUBAÇÃO TRAQUEAL E POPULAÇÃO}

O tempo de intubação traqueal é um fator diretamente relacionado com a eficiência do TEB, ou seja, quanto maior for o tempo da intubação, maior será a probabilidade de ocorrer o laringoespasmo, devido a agressão da laringe e as conseqüentes alterações morfofuncionais. Então, a eficácia do TEB como índice preditor do estridor laríngeo pós-extubação, também aumenta.

Miller e Cole ${ }^{10}$, em estudo quantitativo, com 100 intubações em 88 pacientes com média de tempo de intubação de 5,8 \pm 0,5 dia, encontraram relação entre o resultado do TEB e o aparecimento de estridor laríngeo. Neste estudo, o valor preditivo positivo (VPP) para estridor pós-extubação em pacientes com escape $<110 \mathrm{~mL}$ foi de $80 \%$. Já o valor preditivo para a ausência (VPN) de estridor em pacientes com escape superior a $110 \mathrm{~mL}$ foi de $98 \%$. A especificidade (ESP) e sensibilidade (SEN) observadas foram $99 \%$ e $67 \%$, respectivamente. Neste estudo observou-se que cinco pacientes sofreram extubação acidental, que pode ter contribuído significativamente para o aparecimento de estridor pós-extubação. Portanto, quando estes pacientes foram excluídos da análise, o valor da SEN aumentou de $67 \%$ para $80 \%$. Em estudo realizado por Engoren ${ }^{7}$ com 524 pacientes submetidos à cirurgia cardíaca, foram avaliadas 531 
extubações com tempo médio de ventilação mecânica de $12,9 \mathrm{~h}$ e apenas $5,1 \%$ do total foram ventilados por mais de 48h. Ao contrário de Miller e Cole ${ }^{10}$, Engoren ${ }^{7}$ observou que apenas três pacientes apresentaram estridor laríngeo após as extubações. Porém, todos eles apresentaram escape maior que $300 \mathrm{~mL}$, enquanto nenhum dos 20 pacientes que tiveram escape menor que $110 \mathrm{~mL}$ apresentaram estridor. A SEN e o VPP foram de 0\%, enquanto ESP e o VPN $96 \%$ e $99 \%$, respectivamente. Diante desses resultados, o autor concluiu que apesar do teste ser simples, seguro e de baixo custo, não deve ser utilizado rotineiramente em pacientes submetidos à cirurgia cardíaca, devido ao curto período de ventilação mecânica, comprometendo a sua eficácia.

Sandhu e col. ${ }^{12}$, em um centro de trauma, avaliaram a forma quantitativa do TEB e observaram que dos 100 pacientes incluídos no estudo, apenas 13 evoluíram com laringoespasmo. A média de escape foi de $11,7 \%$, sendo que 7 - com média de escape de $8,4 \%$ do volume-corrente inspiratório programado - apresentaram somente o estridor, enquanto os seis restantes foram re-intubados com média de escape de 9,2\%. Por outro lado, aqueles pacientes que não tiveram estridor laríngeo pós-extubação tinham média de escape de $57 \%$, apresentando diferença significativa ( $p<0,001)$. Observou-se ainda a nítida relação do tempo de intubação com a incidência de estridor, a média de intubação traqueal foi de $5,9 \pm 5$ dias, sendo que os pacientes com mais de três dias de ventilação mecânica tiveram aumento significativo do risco de apresentar estridor.

De Bast e col. ${ }^{13}$ observaram em estudo com o teste de escape quantitativo em 76 pacientes, com média de tempo de intubação de $5 \pm 3$ dias, a incidência de laringoespasmo igual a $11 \%$; com SEN igual a $75 \%$ e ESP de $72,1 \%$, VPP e VPN de $15 \%$ e $96,1 \%$, respectivamente. Maury e col. ${ }^{14}$ obtiveram resultados similares, analisando o teste qualitativamente, com tempo de intubação de 3,5 $\pm 3,4$ dias, sugerindo a não interferência do tipo de teste nos resultados dos estudos. Já Jaber e col. ${ }^{15}$, analisando o teste na forma quantitativa com um total de 120 extubações em 112 pacientes, com média de tempo de intubação $5,5 \pm 6,3$ dias, encontraram SEN e ESP de $85 \%$ e $95 \%$, respectivamente para o teste de escape, com VPP de $69 \%$ e VPN de $99 \%$. Chung e col. ${ }^{4}$, observaram que o TEB obteve resultado satisfatório com 95 pacientes, com média de tempo de intubação de $28,1 \pm 17,6$ dias. Trinta e cinco $(36,8 \%)$ pacientes evoluíram com laringoespasmo grave, apresentando média de escape de 53,9 $\pm 56,2 \mathrm{~mL}$, média muito abaixo daqueles que não apresentaram laringoespasmo $(287,9 \pm 120 \mathrm{~mL}), \mathrm{SEN}=88,6 \%, \mathrm{ESP}=90 \%$, VPP $=83,8 \%$ e VPN $=93,1 \%$.

Sadaka e col. ${ }^{16}$ em estudo com 61 pacientes com média de intubação de 2,6 \pm 2 dias, observaram que apenas quatro pacientes $(6,6 \%)$ desenvolveram estridor pós-extubação. Seis pacientes obtiveram escape inferior a $130 \mathrm{~mL}$, dos quais dois apresentaram estridor, com SEN igual a 50\%. Os autores concluíram que o TEB não pode predizer de forma satisfatória sobre os pacientes com risco de desenvolver estridor pósextubação. Corroborando com Kriner e col. ${ }^{17}$, que 20 $(4,3 \%)$ dos 462 pacientes em seu estudo, sob ventilação mecânica, seja por motivos clínicos ou cirúrgicos por mais de $24 \mathrm{~h}$, apresentaram estridor pós-extubação. Desses, 10 pacientes apresentaram TEB positivo, utilizando como ponto de corte $110 \mathrm{~mL}$ de escape $(\mathrm{SEN}=50 \%$, ESP $=84 \%$, VPP $12 \%$ e VPN 97\%) e quando utilizado $15,5 \%$ do volume-corrente expirado, sete dos 48 pacientes que falharam, apresentaram estridor revelando SEN $=35 \%$, ESP $=91 \%$, VPP $15 \%$ e VPN 97\%. Infere-se deste estudo que o TEB não é um índice confiável para predizer o laringoespasmo, não devendo ser usado como indicação de adiar a extubação ou iniciar outros tratamentos específicos.

Recentemente, Souza e col. ${ }^{18}$ avaliaram um tipo de teste de permeabilidade de vias aéreas com o paciente em ventilação espontânea $(n=20)$ por curto período de tempo em ventilação mecânica; no entanto, não foi observada a presença de laringoespasmo após as extubações. Neste estudo os pacientes que apresentaram valor de escape inferior a $12 \%$ quando realizaram o teste em ventilação espontânea $(n=12 ; 3,97 \% \pm 2,19 \%)$, apresentaram valores bem abaixo quando realizado o teste quantitativo $(n=4 ; 7,27 \% \pm 2,3 \%$ ), fato que pode ser decorrente de estar sem auxílio da ventilação com pressão positiva. Dessa forma, os autores sugerem que o ponto de corte para o teste em pacientes sob ventilação espontânea deva ser muito menor para aqueles em ventilação mecânica (Tabela 1).

Os estudos abordados revelaram importante relação entre o tempo de intubação e a acurácia do TEB, sendo observado que quanto menor o tempo de intubação, menos sensível será o teste. Em pacientes intubados com média de tempo de $48 \mathrm{~h}$ ou mais, seu valor como índice preditivo para estridor é grande. No entanto, para pacientes intubados com tempo igual ou inferior a 48h, principalmente naqueles submetidos à cirurgia cardíaca, o teste é pouco sensível, não devendo ser recomendado a sua utilização como rotina para extubação. 
Tabela 1 - Características dos Estudos em Relação à Aplicação do TEB

\begin{tabular}{|c|c|c|c|c|c|c|c|c|c|c|c|}
\hline \multirow{2}{*}{ Autores } & & \multirow{2}{*}{$\begin{array}{l}\text { População } \\
\text { Amostra }\end{array}$} & \multicolumn{8}{|c|}{ Análise e Resultados } & \multirow[t]{2}{*}{ Comentários } \\
\hline & & & $\begin{array}{l}\text { Tempo de } \\
\text { VM }\end{array}$ & $\begin{array}{l}\text { Ponto } \\
\text { de Corte }\end{array}$ & $\begin{array}{l}\text { Característi- } \\
\text { cas da Popu- } \\
\text { lação }\end{array}$ & Incidência & SEN & ESP & VPP & VPN & \\
\hline $\begin{array}{l}\text { Souza } \\
\text { col. }{ }^{18}\end{array}$ & $\bar{e}$ & $\begin{array}{l}20 \text { extuba- } \\
\text { ções }\end{array}$ & $\begin{array}{c}<2 \text { dias } \\
(80 \%)\end{array}$ & $12 \%$ & $\begin{array}{l}\text { Pacientes em } \\
\text { pós- operató- } \\
\text { rio de cirurgia } \\
\text { cardíaca } \\
(50 \%)\end{array}$ & 0 & * & * & * & * & $\begin{array}{l}\text { Realizado } 3 \text { tipos tes- } \\
\text { tes, consecutivos }\end{array}$ \\
\hline $\begin{array}{l}\text { Chung } \\
\text { col. }^{4}\end{array}$ & e & $\begin{array}{c}95 \text { con- } \\
\text { secutivas } \\
\text { intubações } \\
\text { prolongadas } \\
\text { e pacientes } \\
\text { necessitan- } \\
\text { do traqueos- } \\
\text { tomia eletiva }\end{array}$ & $\begin{array}{l}28 \pm 17,6 \\
\quad \text { dias }\end{array}$ & $\begin{array}{c}38,9 \% \text { ou } \\
140 \mathrm{~mL}\end{array}$ & $\begin{array}{l}\text { IRpA (a } \\
\text { maioria por } \\
\text { pneumonia, } \\
\text { exacerbação } \\
\text { de DPOC) }\end{array}$ & $\begin{array}{c}35 \\
(36,8 \%)\end{array}$ & $88.6 \%$ & $90 \%$ & $83,8 \%$ & $93.1 \%$ & $\begin{array}{l}\text { Foi testada a correla- } \\
\text { ção do TEB com flu- } \\
\text { xo inspiratório, PEEP, } \\
\text { complacência, porém } \\
\text { sem significância }\end{array}$ \\
\hline $\begin{array}{l}\text { Maury } \\
\text { col. }{ }^{14}\end{array}$ & $\mathrm{e}$ & $\begin{array}{l}115 \text { extuba- } \\
\text { ções em } 90 \\
\text { pacientes }\end{array}$ & $\begin{array}{l}3,5 \pm 3,4 \\
\quad \text { dias }\end{array}$ & $\star \star$ & $\begin{array}{l}\text { Intubado } \\
\text { por causas } \\
\text { variadas (6\% } \\
\text { cirúrgico) }\end{array}$ & $\begin{array}{c}4 \\
(3,5 \%)\end{array}$ & $100 \%$ & $80 \%$ & $15 \%$ & $100 \%$ & $\begin{array}{l}\text { Este estudo fez re- } \\
\text { lação do TEB com o } \\
\text { valor de tosse }\end{array}$ \\
\hline
\end{tabular}

\section{IDADE}

O uso do TEB pode se mostrar com acurácia diferente de acordo com a idade dos pacientes estudados. Mhanna e col. ${ }^{19}$ realizaram um estudo com 109 extubações em 105 pacientes separados em dois grupos de crianças: um com idade igual ou inferior a 7 anos, e outro com idade superior a 7 anos (média de idade de 13,3 anos). Nesse estudo evidenciou-se que nos pacientes menores de 7 anos o TEB apresentou baixa sensibilidade para predição de estridor pós-extubação; o contrário pode ser observado em pacientes com idade superior a 7 anos. O que pode ser comparado com o resultado de estudos com a população adulta, onde a idade não influenciou significativamente com o resultado do TEB como índice preditivo para laringoespasmo pós-extubação $7,9,10,13,15,16$.

\section{CONCLUSÃO}

O uso do TEB, na sua forma qualitativa ou quantitativa, pode ser considerado um bom índice preditivo para indicar a presença de estridor laríngeo pós-extubação decorrente de laringoespasmo. Aliado a isso, estudos recentes mostram que a utilização de corticoterapia pré-extubação (e a continuidade de seus efeitos pósextubação), aumentam o valor de escape e reduzem a incidência de estridor em pacientes com alto risco de desenvolver laringoespasmo (volume de escape $<110$ $\mathrm{mL})^{20,21}$. Em geral, o TEB apresenta valores preditivos negativos elevados, o que evidencia importância significativa para a observação de ausência de estridor pósextubação. Pode-se, dessa forma, identificar que quando há o escape, os pacientes desse grupo apresentam pouca probabilidade de desenvolver laringoespasmo. Todavia, a sua confiabilidade é sensivelmente diminuída em pacientes com menos de sete anos, com tempo de intubação inferior a $48 \mathrm{~h}$, e principalmente, em pacientes submetidos à cirurgia cardíaca com tempo de ventilação inferior a 24h. Dessa maneira, observa-se que o TEB se mostra eficaz para a predição do laringoespasmo pós-extubação, tendo o fisioterapeuta em mãos um instrumento simples, de baixo custo e confiável para o momento da extubação. 


\begin{tabular}{|c|c|c|c|c|c|c|c|c|c|c|}
\hline Autores & $\begin{array}{c}\text { População } \\
\text { Amostra }\end{array}$ & $\begin{array}{c}\text { Tempo de } \\
\text { VM }\end{array}$ & $\begin{array}{c}\text { Ponto } \\
\text { de Corte }\end{array}$ & $\begin{array}{l}\text { Característi- } \\
\text { cas da Popu- } \\
\text { lação }\end{array}$ & Incidência & SEN & ESP & VPP & VPN & \\
\hline $\begin{array}{l}\text { Jaber e } \\
\text { col. }{ }^{15}\end{array}$ & $\begin{array}{l}120 \text { extuba- } \\
\text { ções em } 112 \\
\text { pacientes }\end{array}$ & $\begin{array}{l}5,5 \pm 6,3 \\
\quad \text { dias }\end{array}$ & $\begin{array}{l}12 \% \text { ou } \\
130 \mathrm{~mL}\end{array}$ & $\begin{array}{l}76 \% \text { cirúrgi- } \\
\text { cos, } 24 \% \text { de } \\
\text { outras causas }\end{array}$ & $\begin{array}{c}14 \\
(12 \%)\end{array}$ & $85 \%$ & $95 \%$ & $69 \%$ & $98 \%$ & $\begin{array}{l}\text { Bom índice preditivo } \\
\text { para o estridor pos } \\
\text { extubação, quando } \\
\text { associado ao alto va- } \\
\text { lor SAPS II, auto ex- } \\
\text { tubação, dificuldade } \\
\text { ou grande período de } \\
\text { intubação }\end{array}$ \\
\hline $\begin{array}{l}\text { De Bast } \\
\text { e col. }{ }^{13}\end{array}$ & $\begin{array}{c}76 \text { extuba- } \\
\text { ções }\end{array}$ & $5 \pm 3$ dias & $15,5 \%$ & $\begin{array}{l}61 \% \text { cirúrgi- } \\
\text { cos e } 39 \% \\
\text { clínicos }\end{array}$ & $\begin{array}{c}8 \\
(11 \%)\end{array}$ & $75 \%$ & $72.1 \%$ & $25 \%$ & $96.1 \%$ & $\begin{array}{l}\text { O teste se mostrou } \\
\text { como bom índice e } \\
\text { o escape de mais de } \\
15,5 \% \text { pode ser usa- } \\
\text { do para limitar o risco } \\
\text { de reintubação }\end{array}$ \\
\hline $\begin{array}{l}\text { Sandhu e } \\
\text { col. }{ }^{12}\end{array}$ & $\begin{array}{c}110 \text { pacien- } \\
\text { tes }\end{array}$ & $\begin{array}{c}5,9 \pm 5 \\
\text { dias em } \\
\text { pacientes } \\
\text { com estri- } \\
\quad \text { dor }\end{array}$ & $10 \%$ & $\begin{array}{l}\text { Pacientes } \\
\text { politraumati- } \\
\text { zados, na sua } \\
\text { maioria com } \\
\text { TCE }\end{array}$ & $\begin{array}{c}7 \\
(6.4 \%)\end{array}$ & $* \star$ & $99 \%$ & $\star *$ & ** & $\begin{array}{l}\text { Tempo de ventilação } \\
\text { mecânica de } 2.6 \pm \\
2.6 \text { dias em pacientes } \\
\text { sem estridor }\end{array}$ \\
\hline Engoren ${ }^{7}$ & $\begin{array}{l}531 \text { extuba- } \\
\text { ções em } 524 \\
\text { pacientes }\end{array}$ & $\begin{array}{l}12,9 \text { horas } \\
(10,5- \\
21,2 \mathrm{~h})\end{array}$ & $110 \mathrm{~mL}$ & $\begin{array}{l}\text { Pacientes em } \\
\text { pós- operató- } \\
\text { rio de cirurgia } \\
\text { cardíaca }\end{array}$ & $\begin{array}{c}3 \\
(0,6 \%)\end{array}$ & $0 \%$ & $96 \%$ & $0 \%$ & $99 \%$ & $\begin{array}{l}\text { O teste mostrou-se } \\
\text { sem acurácia, não de- } \\
\text { vendo ser recomen- } \\
\text { dado como rotina em } \\
\text { pacientes submetidos } \\
\text { a cirurgia cardíaca }\end{array}$ \\
\hline $\begin{array}{l}\text { Miller e } \\
\text { Cole }^{10}\end{array}$ & $\begin{array}{l}100 \text { extuba- } \\
\text { ções conse- } \\
\text { cutivas em } \\
88 \text { pacientes }\end{array}$ & $\begin{array}{c}5.8 \pm 0.5 \\
\text { dias. }\end{array}$ & $\begin{array}{c}15,5 \% \\
\text { ou } 110 \\
m L\end{array}$ & $\begin{array}{l}\text { IRpA (exceto } \\
\text { pós-operató- } \\
\text { rios, grandes } \\
\text { queimados ou } \\
\text { traumatizados) }\end{array}$ & $\begin{array}{c}6 \\
(6 \%)\end{array}$ & $67 \%$ & $99 \%$ & $80 \%$ & $98 \%$ & $\begin{array}{l}\text { O teste mostrou-se } \\
\text { como bom índice } \\
\text { preditivo para o apa- } \\
\text { recimento do estridor } \\
\text { laríngeo }\end{array}$ \\
\hline
\end{tabular}

* Não se aplica

** Sem referência

\section{REFERÊNCIAS}

01. Epstein S - Predicting extubation failure: is it in (on) the cards? Chest, 2001;120:1061-1063.

02. Meade M, Guyatt G, Cook D et al. - Predicting success in weaning from mechanical ventilation. Chest, 2001;120:(Suppl6):400S-424S.

03. Prinianakis G, Alexopoulou C, Mamidakis E et al - Determinants of the cuff-leak test: a physiological study. Crit Care, 2005;9:R24-R31.

04. Chung $\mathrm{YH}$, Chao TY, Chiu CT et al - The cuff-leak test is a simple tool to verify severe laryngeal edema in patients undergoing long-term mechanical ventilation. Crit Care Med, 2006;34:409-414.

05. Barbosa PM, Santos BM - Morphologic trachea alterations in intubated patients in function of intubation time. Rev Lat Am Enfermagem, 2003;11:727-733.

06. Castilho EC, Braz JRC, Cataneo AJM et al - Efeitos da pressão limite $\left(25 \mathrm{cmH}_{2} \mathrm{O}\right)$ e mínima de "selo" do balonete de tubos traqueais sobre a mucosa traqueal do cão. Rev Bras Anestesiol 2003;53:743-755.

07. Engoren $\mathrm{M}$ - Evaluation of the cuff-leak test in a cardiac surgery population. Chest, 1999;116:1029-1031.

08. Ding LW, Wang HC, Wu HD et al - Laryngeal ultrasound: a useful method in predicting post- extubation stridor. A pilot study. Eur Respir J, 2006;27:384-389.

09. Fisher MM, Raper RF - The 'cuff-leak' test for extubation. Anaesthesia, 1992;47:10-12.

10. Miller RL, Cole RP - Association between reduced cuff leak volume and postextubation stridor. Chest, 1996;110:1035-1040.

11. De Backer D - The cuff-leak test: what are we measuring? Crit Care,

2005;9:31-33.

12. Sandhu RS, Pasquale MD, Miller K et al - Measurement of endotrachea tube cuff leak to predict postextubation stridor and need for reintubation. J Am Coll Surg, 2000;190:682-687.

13. De Bast Y, De Backer D, Moraine JJ et al - The cuff leak test to predict failure of tracheal extubation for laryngeal edema. Intensive Care Med, 2002;28:1267-1272.

14. Maury E, Guglielminotti J, Alzieu M et al - How to identify patients with no risk for postextubation stridor? J Crit Care, 2004;19:23-28.

15. Jaber S, Chanques G, Matecki S et al - Post-extubation stridor in intensive care unit patients: Risk factors evaluation and importance of the cuff-leak test. Intensive Care Med, 2003;29:69-74.

16. Sadaka F, Trottier S, Kane T - The cuff-leak test and postextubation stridor in intensive care unit patients. Crit Care Med, 2004;32:(Suppl12):A119.

17. KrinerEJ, Shafazand S, Colice GL-The endotracheal tube cuff-leak test as predictor for postextubation stridor. Respir Care, 2005;50:1632-1638.

18. Souza SS, Figueiredo LC, Guedes CAV et al - Teste de permeabilidade de vias aéreas pré-extubação: comparação entre três métodos em ventilação espontânea. Rev Bras Ter Intensiva, 2007;19:310-316.

19. Mhanna MJ, Zamel YB, Tichy CM et al - The "air leak" test around the endotracheal tube, as a predictor of postextubation stridor, is age dependent in children. Crit Care Med, 2002;30:2639-2643.

20. Lee $\mathrm{CH}$, Peng MJ, Wu CL - Dexamethasone to prevent postextubation airway obstruction in adults: a prospective, randomized, double-blind, placebo-controlled study. Crit Care, 2007;11:R72.

21. Cheng $\mathrm{KC}$, Hou CC, Huang $\mathrm{HC}$ et al - Intravenous injection of methylprednisolone reduces the incidence of postextubation stridor in intensive care unit patients. Crit Care Med, 2006;34:1345-1350. 